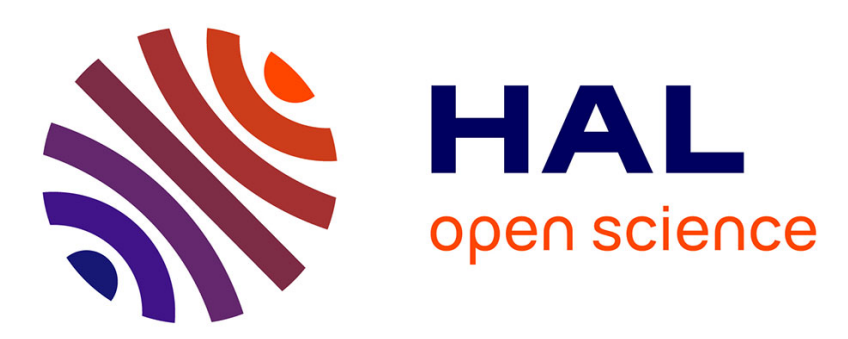

\title{
Problèmes de construction de type polynomial II - Quelques résultats d'existence de plans sphériques isovariants exacts
}

Frédéric Bertrand

\section{- To cite this version:}

Frédéric Bertrand. Problèmes de construction de type polynomial II - Quelques résultats d'existence de plans sphériques isovariants exacts. 2008. hal-00278267v2

\section{HAL Id: hal-00278267 \\ https://hal.science/hal-00278267v2}

Preprint submitted on 12 May 2008

HAL is a multi-disciplinary open access archive for the deposit and dissemination of scientific research documents, whether they are published or not. The documents may come from teaching and research institutions in France or abroad, or from public or private research centers.
L'archive ouverte pluridisciplinaire HAL, est destinée au dépôt et à la diffusion de documents scientifiques de niveau recherche, publiés ou non, émanant des établissements d'enseignement et de recherche français ou étrangers, des laboratoires publics ou privés. 


\title{
Problèmes de construction de type polynomial II - Quelques résultats d'existence de plans sphériques isovariants exacts
}

\author{
FRÉDÉRIC BERTRAND* \\ Université Louis Pasteur \\ Institut de Recherche Mathématique Avancée ${ }^{\dagger}$
}

11 mai 2008

\begin{abstract}
Résumé
En utilisant l'algèbre computationnelle, plus particulièrement les bases de Gröbner, nous résolvons des problèmes de construction de type polynomial et en déduisons un théorème d'existence de plans isovariants sphériques dont les coordonnées des points support sont connues exactement dans $\mathbb{R}^{3}$ ce qui permet l'utilisation de la statistique algébrique pour obtenir une détermination complète de leurs confusions d'effets. L'intérêt de ces plans est multiple : ils sont utilisables, par exemple, pour l'étude des surfaces de réponse et des formes tri-dimensionnelles.
\end{abstract}

\begin{abstract}
Polynomial designs II - Some existence results of exact rotatable spherical designs

Using computational commutative algebra, especially Gröbner bases, we first find out several polynomial designs and then state existence results in $\mathbb{R}^{3}$ for spherical rotatable designs whose coordinates of their support points are known exactly, thus enabling us to derive the ideal of confounding polynomials thanks to tools defined in the framework of algebraic statistics. These designs can be of use in various settings such as response surface methodology or tridimensional shape analysis.
\end{abstract}

\section{Abridged English version}

In this paper, we consider an experimental domain $\chi$ which is a closed ball whose center is the origin of an Euclidean vector space of dimension $v=3$. We set, in section 3 , the classical statistical framework of full polynomial regression models of degree $d$ over the experimental domain $\chi$ as defined by equations (1) and $(2)$.

In the preprint, Ber08b, we showed that the search for spherical rotatable

\footnotetext{
*Courriel : fbertran@math.u-strasbg.fr

$\dagger 7$ rue René Descartes, 67084 Strasbourg Cedex
} 
designs, BH57, can be stated in the general framework of the construction of polynomial designs. We successfully derived designs in $\mathbb{R}^{3}$ using the methodology highlighted in Ber08b and a preliminary study of Hardin and Sloane, HS96, which leads to theorems 4.1 and 4.2 .

The first one of the two main benefits of this achievement is that the coordinates of the points of all these designs are known exactly, thus enabling us to use the tools arising in algebraic statistics, PRW00, and for example finding out all the confoundings or performing lack of fit tests. As a consequence we provide designs of many different sizes all featuring not only a praised property, rotatability, but also, for the first time, state of the art ways to analyse them.

The second one is that not only the designs for which we exactly computed the coordinates of the points supporting the design but also all the designs in the list given by Hardin and Sloane in [HS96] are of major interest in three-dimensional shape analysis. Indeed, the theorem 5.2 states that they are, when one uses a model defined as a free finite family of spherical harmonics, orthogonal up to a known degree. They are also optimal for any of the Kiefer's $\Phi_{p}$ criteria, [Kie74, according to a result of Dette, Melas and Pepelyshev, [DMP05, for a full model of spherical harmonics of the same degree, see table 1 for more details. We lay the stress on fact that three-dimensional shape analysis is of use in many different domains such as medecine, chemistry, architecture, agriculure and biology.

Such a work is to be carried out in dimension 4, as soon as the preliminary study of Sloane, Hardin and Cara, SHC03, will be completed.

\section{Motivation}

Dans cette note nous nous intéressons à une série de dispositifs qui ont été construits ou étudiés par Hardin et Sloane dans leur article [HS96]. Hardin et Sloane ont mis en libre disposition les coordonnées approchées des points support de ces plans sphériques combinatoires. Toutefois, comme les coordonnées des points support du plan ne sont pas connues exactement, ces informations ne permettent pas d'utiliser les techniques de statistique algébrique développées par Pistone, Riccomagno et Wynn, [PRW00], dont il est particulièrement judicieux de pouvoir se servir pour déterminer complètement les nombreuses confusions d'effets qui apparaissent naturellement puisque le support de ces plans sphériques combinatoires est inclus dans la sphère unité. En ajoutant un essai au centre du domaine expérimental, nous obtenons des dispositifs qui sont d'un intérêt particulier en planification expérimentale, en méthodologie des surfaces de réponse par exemple, puisqu'ils permettent non seulement de construire des plans $\mathcal{G}$-faiblement invariants, pour $\mathcal{G}$ un groupe de matrices compact quelconque, Ber08a, mais sont également exacts, admissibles et isovariants pour des modèles polynomiaux de degré 2 sur un domaine expérimental pris égal à la boule unité de $\mathbb{R}^{3}$. Réciproquement, le support de tout plan admissible et $\left\{I_{3},-I_{3}\right\}$-faiblement invariant est nécessairement l'union d'un point au centre du domaine expérimental et d'un ensemble de points appartenant à la sphère unité, GH96. Si nous considérons un plan dont le support se décompose ainsi, et pour un nombre total d'essai fixé, la $D$-efficacité, [BH57], [Kie60] et [NS92], et la $I$-efficacité, HS92 et HS95, du plan est maximisée s'il est possible d'organiser la partie sphérique du plan comme un plan sphérique combinatoire de 
force 4. Il est donc primordial de mettre à la disposition des expérimenteurs des constructions de telles constructions pour le plus de cardinaux différents posibles.

Dette, Melas et Pepelyshev, DMP05], font l'étude des plans optimaux pour l'analyse de formes tri-dimensionnelles à l'aide d'harmoniques sphériques. Les domaines d'application de l'acquisition et de l'analyse de formes tri-dimensionnelles sont nombreux et variés : la médecine, la chimie, l'architecture, l'agriculture et la biologie. Il s'agit de parvenir à décrire la valeur de la distance entre l'origine du repère et celle l'extrémité de l'objet dans une direction donnée. Les modèles de régression basés sur des harmoniques sphériques permettent généralement de décrire de manière satisfaisante les formes de ces objets à l'aide d'un nombre relativement réduit de termes. Nous nous plaçons dans le cas où l'expérimentateur est à même de fixer les directions pour lesquelles il effectue un relevé de la distance entre l'origine du repère et celle l'extrémité de l'objet. Il est alors d'un intérêt primordial de déterminer de bons plans expérimentaux dont le support est inclus dans la sphère unité de $\mathbb{R}^{3}$. Dette, Melas et Pepelyshev ont montré que la distribution uniforme sur la sphère est optimale pour tous les critères d'optimalité $\Phi_{p}$ proposés par Kiefer en 1974, [Kie74]. Cette distribution n'étant pas un plan expérimental, les auteurs proposent des plans dont le support est inclus dans la sphère unité de $\mathbb{R}^{3}$. La méthodologie de construction que nous avons proposée dans [Ber08b] permet d'obtenir des plans de cardinaux beaucoup plus variés qui vérifient cette proporiété puisqu'un dispositif isovariant pour un modèle polynomial complet de degré $d$ est orthogonal lorsqu'analysé à l'aide d'un modèle de régression dont les termes sont des harmoniques sphériques de degré inférieur ou égal à $d$.

Les plans obtenus dans cette note sont donc d'un intérêt triple. Premièrement, ce sont des plans isovariants admissibles et de $D$-efficacité et $I$-efficacité maximale parmi des plans dont le support est l'union d'un point au centre du domaine expérimental et d'une partie sphérique. Deuxièmement, ils permettent de construire des plans $\mathcal{G}$-faiblement invariants, pour $\mathcal{G}$ un groupe de matrices compact quelconque, Ber08a. Troisièmement ils peuvent être utilisés avec profit lors de la reconnaissance de formes tri-dimensionnelles puisqu'ils sont optimaux pour tous les critères d'optimalité $\Phi_{p}$. Enfin les coordonnées des points support du plan sont connues exactement, ce qui permet, pour la première fois, d'utiliser les outils de la statistique algébrique pour analyser finement leurs propriétés.

Pour obtenir des résultats explicites de constructions, nous introduisons des problèmes de construction de type polynomial, définis dans Ber08b, et les bases de Gröbner, KR00] et KR05, pour les résoudre.

\section{Notations et définitions}

Nous nous intéressons à des modèles de régression linéaire soumis aux hypothèses statistiques usuelles. Nous considérons une variable explicative $\boldsymbol{x}$, dont les différentes valeurs possibles appartiennent à un domaine expérimental $\chi$, qui est une partie compacte d'un espace vectoriel de dimension finie $\mathbb{R}^{v}, v \in \mathbb{N}$ muni de sa structure euclidienne canonique, dont nous étudions l'influence sur une réponse $y$ à valeurs réelles. Soient $d$ un entier naturel et $A_{d}$ le sous-ensemble fini de $\mathbb{N}^{v}$ défini par $A_{d}=\left\{\boldsymbol{\alpha} \in \mathbb{N}^{v},|\boldsymbol{\alpha}| \leqslant d\right\}$, où $|\boldsymbol{\alpha}|$ désigne la somme des composantes du vecteur $\boldsymbol{\alpha}$. Les éléments de $A_{d}$ sont notés $\boldsymbol{\alpha}=\left(\alpha_{1}, \ldots, \alpha_{v}\right)$. Nous 
posons $k=\operatorname{Card}\left(A_{d}\right)=C_{d+v-1}^{v-1}$ et, pour $\boldsymbol{x} \in \mathbb{R}^{v}, \boldsymbol{f}(\boldsymbol{x})=\left(\boldsymbol{x}^{\boldsymbol{\alpha}}\right)_{\boldsymbol{\alpha} \in A_{d}}$ avec $\boldsymbol{x}^{\left(\alpha_{1}, \ldots, \alpha_{v}\right)}=x_{1}^{\alpha_{1}} \times \cdots \times x_{v}^{\alpha_{v}}$. Un modèle de régression multiple polynomiale complet de degré $d$, noté $A_{d}$, sur un domaine expérimental $\chi \in \mathbb{R}^{v}$ est alors défini par :

$$
y(\boldsymbol{x})=\boldsymbol{\theta}^{\prime} \boldsymbol{f}(\boldsymbol{x})=\sum_{\boldsymbol{\alpha}=\left(\alpha_{1}, \ldots, \alpha_{v}\right) \in A_{d}} \theta_{\boldsymbol{\alpha}} \prod_{i=1}^{v} x_{i}^{\alpha_{i}}, \quad \forall \boldsymbol{x}=\left(x_{1}, \ldots, x_{v}\right)^{\prime} \in \boldsymbol{\chi}
$$

où $\boldsymbol{\theta}^{\prime}=\left(\theta_{\boldsymbol{\alpha}}\right)_{\boldsymbol{\alpha} \in A_{d}} \in \mathbb{R}^{k}$, est un vecteur de paramètres inconnus. Il s'agit de la part déterministe d'un modèle stochastique défini de la manière suivante : l'observation des valeurs de la réponse $y$ aux points $\boldsymbol{x}_{1}, \ldots, \boldsymbol{x}_{\boldsymbol{n}} \in \boldsymbol{\chi}$ est représentée par des variables aléatoires à valeurs réelles $Y_{1}, \ldots, Y_{n}$ telles que

$$
\begin{array}{rl}
i=1, \ldots, n & \mathbb{E}\left[Y_{i}\right]=y\left(\boldsymbol{x}_{\boldsymbol{i}}\right), \quad \operatorname{Var}\left[Y_{i}\right]=\sigma^{2}, \\
i, j=1, \ldots, n, i \neq j & \mathbb{C} \operatorname{Cov}\left[Y_{i}, Y_{j}\right]=0 .
\end{array}
$$

La variance constante $\left.\sigma^{2} \in\right] 0,+\infty[$ est inconnue et est de ce fait un paramètre additionnel du modèle.

Nous supposons que les valeurs pour lesquelles les observations sont effectuées sont connues exactement et contrôlées par l'expérimentateur : il s'agit du contexte usuel de la planification expérimentale.

Définition 3.1. Un plan $\boldsymbol{\xi}$ approché pour le modèle défini par les équations (1) et (2) est une mesure de probabilité de support fini sur $\boldsymbol{\chi}$, c'est-à-dire un couple $(\boldsymbol{X}, \boldsymbol{w}(\boldsymbol{X}))$ où $\boldsymbol{X}$ est un ensemble fini de $r$ points $\left\{\boldsymbol{x}_{\mathbf{1}}, \ldots, \boldsymbol{x}_{\boldsymbol{r}}\right\} \in \boldsymbol{\chi}$, le support du plan, $\boldsymbol{w}=\left(w_{1}\left(\boldsymbol{x}_{\mathbf{1}}\right), \ldots, w_{r}\left(\boldsymbol{x}_{\boldsymbol{r}}\right)\right)$ sont les poids des points support du plan. Soit $n$ le nombre total d'essais que nous souhaitons effectuer. Un plan approché $\boldsymbol{\xi}$, ne peut être réalisé dans la pratique que s'il existe $r$ entiers strictement positifs $n_{1}, \ldots, n_{r}$ de somme $n$ tels que $\boldsymbol{\xi}\left(\boldsymbol{x}_{\boldsymbol{i}}\right)=n_{i} / n$ pour $i=1, \ldots, r$. Un tel plan est appelé plan exact de taille $n$ et nous le notons alors $\boldsymbol{\xi}_{\boldsymbol{n}}$.

Tous les plans proprosés dans la suite sont exacts puisque les poids sont tous égaux. La propriété d'amissibilité ne dépendant pas des poids du plan, leur support permet d'obtenir des plans admissibles pour les configurations de poids possibles.

\section{Plans isovariants dans $\mathbb{R}^{3}$}

La construction des dispositifs exacts détaillée dans [Ber07] a comme première conséquence les théorèmes suivants sur l'existence de plans isovariants dont les coordonnées des points support sont connues de manière exacte pour un modèle de degré 2 .

Théorème 4.1. Il existe pour $N \in\{13,15\} \cup[\mid 17,+\infty[\mid$ des plans de cardinal $N$ qui, analysés avec un modèle polynomial complet de degré 2 , sont isovariants. Le support de chacun des plans est l'union d'un plan sphérique et d'un point au centre du domaine expérimental. Dans tous les cas, ils peuvent ne comporter aucune répétition dans leur partie sphérique et en fonction du cardinal du plan recherché un nombre partiel ou total de répétitions. 
Pour chacune des valeurs de $N$ suivantes 13, 15, 17, 18, 19, 20, 21, 22, 23, 24, 25, 26, 28, 29, 31, 33, 37, 39, 49, 55, 57, 61, 73, 81, les coordonnées exactes de ces plans sont connues et ces plans ne sont pas construits comme l'union de deux plans de cardinal inférieur.

\section{Démonstration}

L'existence et le calcul des coordonnées exactes des plans sphériques de force supérieure ou égale à 4 lorsque $N$ est égal à $12,14,16,17,18,19,20,21,22,23$, $24,25,27,28,30,32,36,38,48,54,56,60,72,80$ découlent de l'étude de cas réalisée dans [Ber07. L'ajout d'un point au centre à ces dispositifs les rend donc isovariants lorsqu'ils sont analysés par un modèle de degré 2 et nous obtenons les valeurs de cardinaux mentionnées dans le théorème ci-dessus. Ce qui prouve la seconde partie du théorème.

Rappelons que l'union de deux plans sphériques de force $t$ est un plan sphérique de force $t$. Ainsi pour tout cardinal $N$ pouvant s'écrire comme $1+N_{1}+N_{2}+$ $\ldots+N_{k}$ où $N_{1}, N_{2}, \ldots, N_{k}$ sont $k$ entiers de la liste $12,14,16,17,18,19$, $20,21,22,23,24,25,27,28,30,32,36,38,48,54,56,60,72,80$ sont des plans isovariants pour un modèle de degré 2. En particulier si nous disposons d'une suite de 12 valeurs consécutives $C_{1}, \ldots, C_{12}$ pour lesquelles il existe un tel dispositif alors en ajoutant le nombre convenable de fois un plan sphérique de cardinal 12, l'icosaèdre, nous pourrons obtenir des plans isovariants pour un modèle d'ordre pour tous les cardinaux supérieurs ou égaux à $C-1$.

Considérons la suite de valeurs 17, 18, 19, 20, 21, 22, 23, 24, 25, 26, 28. Il manque la valeur 27 . Or $27=1+12+14$, ainsi il existe un plan isovariant de cardinal 27 pour un modèle d'ordre 2 . Nous le construisons comme l'union d'un plan sphérique de cardinal 12 de force supérieure à 4, d'un plan sphérique de cardinal 14 de force supérieure à 4 et d'un point au centre. Nous obtenons alors 12 valeurs consécutives de 17 à 28 , ce qui donne l'existence pour $N \in\{13,15\} \cup[\mid 17,+\infty[\mid$. Ces plans peuvent comporter des répétitions.

Nous raffinons la construction précédente de la manière suivante : l'image d'un plan sphérique par une transformation orthogonale est un plan sphérique. Nous éviterons ainsi les répétitions en transformant les dispositifs dont nous prenons l'union par une rotation. Reste à trouver une famille infinie de rotations dont les coefficients de la matrice sont algébriques. Ce problème se réduit à savoir s'il existe une famille infinie $\Theta$ de valeurs de $\theta$ pour lesquelles $\cos (\theta)$ est algébrique. Nous pouvons aborder ce problème à l'aide de la théorie des polygones réguliers à $n$ côtés constructibles à la règle et au compas. En effet leurs coordonnées dans un repère s'expriment alors comme $(\cos (2 \pi * k / n), \sin (2 \pi * k / n))$. Si un polygone régulier à $n$ côtés est constructible à la règle et au compas alors $\cos (2 \pi * k / n)$ et $\sin (2 \pi * k / n)$ sont algébriques sur $\mathbb{Q}$ et leurs degrés sont une puissance de 2. Il existe une famille infinie de tels polygones, ceux pour lesquels nous avons $n=2{ }^{i} F_{1}^{j_{1}} F_{2}^{j_{2}} \ldots F_{k}^{j_{k}}$ où $i$ est un entier naturel supérieur à 1 et pour $p \in[|1, k|]$, $j_{p}=0$ ou $j_{p}=1$ et $F_{j}$ sont les nombres premiers de Fermat c'est-à-dire les nombres premiers de la forme $2^{2^{j}}+1$, voir par exemple Car01. Puisqu'il existe des nombres de Fermat premiers, par exemple 3, 5, 17, 257 et 65537, il existe bien une infinité de polygones réguliers dont les coordonnées sont algébriques.

Conjecture 4.1. Hardin et Sloane 1996, [?]

La valeur, conjecturée comme égale à 3 par Hardin et Sloane, de $\tau(13)$ et $\tau(15)$ 
permet de formuler la conjecture suivante : il n'existe pas de dispositif, union d'un plan sphérique et d'un point au centre et de cardinal 14 et 16, qui soit isovariant s'il est analysé à l'aide d'un modèle polynomial de degré 2.

Théorème 4.2. Pour $N \in[\mid 13,+\infty[\mid$, il existe des plans de cardinal $N$, dont les coordonnées des points support du plan sont connues exactement, et qui sont isovariants s'il sont analysés avec un modèle polynomial de degré 2.

Précisons la nature de chacun de ces dispositifs :

- Le support de chacun d'entre eux est l'union d'un plan sphérique et d'au moins un point au centre. Il est possible de spécifier le nombre total d'essais $N$ et le nombre $k \geqslant 1$ de répétitions au centre du domaine arbitrairement dès que $N \geqslant k+16, N=k+14$ ou $N=k+12$. Les valeurs de $N$ et de $k$ pour lesquelles nous avons $N=k+15$ ou $N=k+13$ ne sont pas accessibles, il suffit de prendre à la place de la valeur $k$ la valeur $k^{\prime}=k+1$ ou la valeur $k^{\prime \prime}=k-1$.

- Pour toutes les valeurs de $N$ possibles, il est possible de construire des dispositifs ne comportant aucune répétition hormis à l'origine du repère.

- En fonction de la valeur de $N$, il est possible de construire des dispositifs possédant un nombre partiel ou total de répétitions dans la partie sphérique du dispositif.

\section{Démonstration}

Puisque nous autorisons plus d'une répétition au centre du domaine, les cardinaux de 14 et 16 deviennent accessibles : $14=12+2,16=12+4=14+2$ ce qui prouve le premier point du théorème.

Si $N \geqslant k+16, N=k+14$ ou $N=k+12$, le théorème 4.1 précédent indique qu'il existe un plan isovariant pour un modèle de degré construit comme l'union d'un plan sphérique de cardinal $N-k$ et d'un point au centre. L'ajout d'essais au centre ne modifiant pas le caractère isovariant d'un dispositif, un dispositif formé d'un plan sphérique de cardinal $N-k$ et de $k$ points au centre est isovariant lorsqu'il est analysé à l'aide un modèle d'ordre 2. Si $N=13$ ou $N=15$, le changement de $k$ en $k^{\prime}$ ou en $k^{\prime \prime}$ découle des mêmes considérations.

Signalons enfin, que les plans construits ici, héritent, pour leur partie sphérique, des propriétés des plans construits au théorème 4.1, en particulier la possibilité de les obtenir sans répétitions dans leur partie sphérique.

La situation se complique lorsque nous considérons des plans analysés par des modèles de degré supérieur ou égaux à trois en raison des confusions d'effets qui sont plus complexes à éliminer. En effet il faut alors ajouter d'autres points que des points au centre du domaine ce qui modifiera la fonction génératrice des moments du plan et affectera la propriété d'isovariance.

Il existe plusieurs manières de traiter cette difficulté. L'approche par les $t$-mesures euclidiennes est une possibilité pour obtenir des dispositifs isovariants. Une autre manière de remédier à cette situation est de considérer des modèles qui sont naturellement adaptés au contexte sphérique. 


\section{Plans optimaux pour la reconnaissance de for- mes tri-dimensionnelles}

Nous introduisons pour cela les fonctions polynomiales définies sur la sphère unité, pour plus de détails voir [CLO96.

Proposition 5.1. Les fonctions polynomiales définies sur la sphère unité sont isomorphes à l'anneau quotient $M_{\mathcal{S}}=\mathbb{R}[\boldsymbol{x}] /\left(X^{2}+Y^{2}+Z^{2}-1\right)$, nous notons $M_{\mathcal{S}, k}=\mathbb{R}_{k}[\boldsymbol{x}] /\left(X^{2}+Y^{2}+Z^{2}-1\right)$. Une base de $M_{\mathcal{S}, k}$ est constituée par la somme directe orthogonale de l sous-espaces vectoriels chacun engendré par une base orthonormale de polynômes harmoniques de degré $l$, pour $0 \leqslant l \leqslant k$.

Définition 5.1. Nous appelons modèle polynomial sphérique de degré d, un modèle pour lequel, avec les notations de [Ber08a], $\operatorname{Vect}(\boldsymbol{f}(\mathcal{S}))=M_{\mathcal{S}, d}$.

Théorème 5.1. Si, à la place du modèle polynomial classique d'ordre $k$, où $k$ est un entier supérieur ou égal à 1, nous considèrons le modèle polynomial sphérique pour analyser un dispositif expérimental sphérique, alors il n'y a plus aucune confusion d'effets systématique liée à la nature du domaine expérimental considéré : quelque soit le sous-ensemble de fonctions polynomiales linéairement indépendantes sur la sphère, il existe un plan sphérique pour lequel tous les termes de ce modèle sont estimables.

\section{Démonstration}

Soit $d$ le degré maximal des termes du modèle. Le théorème non constructif de Seymour et Zaslavsky, [SZ84], sur l'existence des plans sphériques de force arbitraire et donc en particulier de force $2 d$. Or la preuve de l'inégalité sur le nombre minimal de points d'un plan sphérique de force $2 d$, voir [DGS77, implique qu'un tel plan rend estimable tous les paramètres associés au modèle sphérique d'ordre $k$.

Proposition 5.2. Les fonctions polynomiales définies sur la sphère unité sont isomorphes à l'anneau quotient $M_{\mathcal{S}}=\mathbb{R}[\boldsymbol{x}] /\left(X^{2}+Y^{2}+Z^{2}-1\right)$, nous notons $M_{\mathcal{S}, k}=\mathbb{R}_{k}[\boldsymbol{x}] /\left(X^{2}+Y^{2}+Z^{2}-1\right)$. Une base de $M_{\mathcal{S}, k}$ est constituée par la somme directe orthogonale de $k+1$ sous-espaces vectoriels chacun engendré par une base orthonormale de polynômes harmoniques de degré $l$, pour $0 \leqslant l \leqslant k$.

La caractérisation des plans sphériques à l'aide des polynômes harmoniques, [Ven01, et l'un des résultats de Dette, Melas et Pepelyshev, DMP05, conduit au théorème 5.2 suivant qui concerne dans un premier temps tous les dispositifs mentionnés dans [HS96, puis, dans le tableau 1] ceux pour lesquels nous avons obtenu les coordonnées exactes des points support du plan. Ces plans ont des cardinaux plus variés que ceux initialement proposés par Dette, Melas et Pepelyshev, [DMP05], ce qui les rend donc d'un intérêt pratique direct.

Théorème 5.2. Les dispositifs de cardinal $N=4,6$ à 100 sont des dispositifs orthogonaux lorsqu'ils sont analysés une famille libre de $M_{\mathcal{S}, 1}$. Ils sont optimaux pour un modèle formé par une base de $M_{\mathcal{S}, 1}$ pour tous les critères d'optimalité $\Phi_{p}$.

Les dispositifs de cardinal $N=12,14,16 \grave{a} 100$ sont des dispositifs orthogonaux 


\begin{tabular}{||c|c||}
\hline \hline Degré & Cardinal du plan $N$ \\
\hline \hline 1 & $4,6,7,8,9,10,11,12,13,14,15,16,17,18,19,20,21$, \\
& $22,23,24,25,27,28,30,32,36,38,48,54,56,60,72,80$. \\
\hline 2 & $\begin{array}{c}12,14,16,17,18,19,20,21,22,23,24,25,27,28,30,32, \\
\end{array}$ \\
\hline 3 & $24,26,27,28,38,48,54,56,59,72,80$. \\
\hline 4 & $36,48,54,56,58,48,54,56,59,72,80$. \\
\hline 5 & $60,72,80$. \\
\hline \hline
\end{tabular}

TAB. 1 - Plans sphériques orthogonaux exacts et optimaux pour un modèle formé par toutes les des harmoniques sphériques d'ordre inférieur ou égal à 1 pour tous les critères d'optimalité $\Phi_{p}$

lorsqu'ils sont analysés avec une famille libre de $M_{\mathcal{S}, 2}$. Ils sont optimaux pour un modèle formé par une base de $M_{\mathcal{S}, 2}$ pour tous les critères d'optimalité $\Phi_{p}$. Les dispositifs de cardinal $N=24,26,28$ à 100 sont des dispositifs orthogonaux lorsqu'ils sont analysés avec une famille libre de $M_{\mathcal{S}, 3}$. Ils sont optimaux pour un modèle formé par une base de $M_{\mathcal{S}, 3}$ pour tous les critères d'optimalité $\Phi_{p}$.

Les dispositifs de cardinal $N=36,40,42,44$ à 100 sont des dispositifs orthogonaux lorsqu'ils sont analysés avec une famille libre de $M_{\mathcal{S}, 4}$. Ils sont optimaux pour un modèle formé par une base de $M_{\mathcal{S}, 4}$ pour tous les critères d'optimalité $\Phi_{p}$.

Les dispositifs de cardinal $N=60,62,64$ à 100 sont des dispositifs orthogonaux lorsqu'ils sont analysés avec une famille libre de $M_{\mathcal{S}, 5}$. Ils sont optimaux pour un modèle formé par une base de $M_{\mathcal{S}, 5}$ pour tous les critères d'optimalité $\Phi_{p}$. Les dispositifs de cardinal $N=84,86$ à 100 sont des dispositifs orthogonaux lorsqu'ils sont analysés avec une famille libre de $M_{\mathcal{S}, 6}$. Ils sont optimaux pour un modèle formé par une base de $M_{\mathcal{S}, 6}$ pour tous les critères d'optimalité $\Phi_{p}$.

Le tableau 1 indique les plans dont les coordonnées exactes sont connues ainsi que le degré jusqu'auquel les propriétés d'orthogonalité et de $\Phi_{p}$-optimalité sont vérifiées.

\section{Conclusion et perspectives}

Cette étude d'un cas particulier de problèmes de construction de type polynomial a montré que la méthodologie de résolution, basée sur l'utilisation des groupes de Coxeter et d'algorithes mettant en jeu les bases de Gröbner et introduite dans la prépublication [Ber08b], permet d'aboutir à la construction de plans expérimentaux dont les coodonnées sont connues de manière exacte. Nous avons alors montré l'existence et indiqué comment obtenir de tels dispositifs pour tous les cardinaux pour lesquels il existe des plans isovariants pour des modèles polynomiaux complets de degré 2 en dimension 3 . Nous en avons également tiré l'existence de dispositifs orthogonaux et $\Phi_{p}$-optimaux pour des problèmes de régression sphérique dont le modèle est spécifié en termes d'harmoniques sphériques d'ordre inférieur ou égal à $1,2,3,4,5$ ou 6 dans le cas de la dimension 3. Ces dispositifs ont une application particulièrement intéressante dans les problèmes de reconnaissance de formes tri-dimensionnelles qui 
sont communs à de nombreux domaines d'application de la planification.

Un prochain article s'intéressera au cas de la dimension 4, dès que l'étude préliminaire en cours de réalisation par Sloane, Hardin et Cara, [SHC03, sera achevée.

\section{Références}

[Ber07] F. Bertrand. Plans sphériques de force $t$ et applications en statistique. Phd thesis, Université Louis Pasteur - Strasbourg I, 2007. http ://tel.archives-ouvertes.fr/tel-00188330/fr/.

[Ber08a] F. Bertrand. Isovariance et $\mathcal{G}$-invariance faible en planification expérimentale. Prépublication de l'IRMA, 2008. http ://hal.archives-ouvertes.fr/hal-00276011/fr/.

[Ber08b] F. Bertrand. Problèmes de construction de type polynomial I - Caractérisations polynomiales des propriétés usuelles d'un plan. Prépublication de l'IRMA, 2008. http ://hal.archives-ouvertes.fr/hal-00277192/fr/.

[BH57] G.E.P. Box and J.S. Hunter. Multi-factor experimental designs for exploring response surfaces. Annals of Mathematical Statististics, $28: 195-241,1957$.

[Car01] J.-C. Carréga. Théorie des corps. La règle et le compas. Hermann, Paris, 2001.

[CLO96] D. Cox, J. Little, and D. O'Shea. Ideals, Varieties and Algorithms : An introduction to Computationnal Algebraic Geometry and Commutative Algebra. Springer-Verlag, New York, 2nd edition, 1996.

[DGS77] P. Delsarte, J.M. Goethals, and J.J. Seidel. Spherical codes and designs. Geometricae Dedicata, 6 :363-388, 1977.

[DMP05] H. Dette, V.B. Melas, and A. Pepelyshev. Optimal designs for threedimensional shape analysis with spherical harmonic descriptors. Annals of Statistics, 33 :2758-2788, 2005.

[GH96] N. Gaffke and B. Heiligers. Approximate designs for polynomial regression : Invariance, admissibility and optimality. In S. Ghosh and C.R. Rao, editors, Handbook of Statistics, volume 13, chapter 30, pages 1149-1199. Elsevier Science B.V., 1996.

[HS92] R.H. Hardin and N.J.A. Sloane. New spherical 4-designs. Discrete Mathematics, 106/107:255-264, 1992.

[HS95] R.H. Hardin and N.J.A. Sloane. Codes (spherical) and (designs) experiments. In Proceedings of Symposia in Applied Mathematics, volume 50, pages 179-206. American Mathematical Society, 1995.

[HS96] R.H. Hardin and N.J.A. Sloane. Mclaren's improved snub cube and other new spherical designs in three dimensions. Discrete Compu. Geom., 15 :429-441, 1996.

[Kie60] J. Kiefer. Optimum experimental designs v, with applications to systematic and rotatable designs. In Proc. 4th Berkeley Sympos. Math. Statist. and Probability, volume 1, pages 381-405, Calif., 1960. Univ. Calif. Press. 
[Kie74] J. Kiefer. General equivalence theory for optimum designs (approximate theory). Annals of Statistics, $2: 849-879,1974$.

[KR00] M. Kreuzer and L. Robiano. Computational Commutative Algebra 1. Springer, Berlin-Heidelberg, 2000.

[KR05] M. Kreuzer and L. Robiano. Computational Commutative Algebra 2. Springer, Berlin-Heidelberg, 2005.

[NS92] A. Neumaier and J.J. Seidel. Measures of strength $2 e$, and optimal designs of degree e. Sankhyā, $54: 299-309,1992$. Dedicated to the memory of R.C. Bose.

[PRW00] G. Pistone, E. Riccomagno, and H.P. Wynn. Gröbner basis methods for structuring and analysing complex industrial experiments. International Journal of Reliability, Quality and Safety Engineering, $7: 285-300,2000$.

[SHC03] N.J.A. Sloane, R.H. Hardin, and P. Cara. Sperical designs in four dimensions. Extended Abstract, 2003.

[SZ84] P.D. Seymour and T. Zavlasky. Averaging sets : A generalisation of mean values and sherical designs. Advances in Mathematics, $52: 213$ $240,1984$.

[Ven01] B Venkov. Réseaux et designs sphériques. In J. Martinet, editor, Réseaux euclidiens, designs sphériques et formes modulaires: Autour des travaux de Boris Venkov, volume 37 of Monographies de L'Enseignement Mathématique, chapter 1. L'enseignement mathématique, Genève, 2001. 